\section{OTU-018 ENDOSCOPIC ULTRASOUND FINE NEEDLE BIOPSY IS SUPERIOR TO FNA FOR ASSESSING PANCREATIC NEUROENDOCRINE TUMOURS}

John Leeds*, Manu Nayar, Sarah Johnson, Colin Wilson, Noor Bekkali, Beate Haugk, Antony Darne, Kofi Oppong. Newcastle Upon Tyne Teaching Hospitals NHS Foundation Trust, Newcastle Upon Tyne, UK

\subsection{6/gutjnl-2018-BSGAbstracts.304}

Introduction Pancreatic neuroendocrine tumours (PanNET) are a distinct tumour type with outcomes dependent, in part, upon grading by Ki67. Endoscopic ultrasound (EUS) guided fine needle aspiration (FNA) shows variable accuracy to determine Ki67 or grading. Our aim was to assess whether Ki67 and grade can be more accurately determined using fine needle biopsy (FNB) compared to FNA using surgical excision histology as the gold standard.

Methods Retrospective analysis of all pancreatic pathology for neuroendocrine tumours was performed for the period Jan 2009 - Jun 2017. Patients were included if they had undergone EUS guided sampling of the lesion prior to surgical resection. Patient demographics, lesion size and location were noted. FNA and FNB results were examined and Ki67 and grade recorded. Surgical histology reports were examined and time from EUS to surgery, operation performed, Ki67 and grade recorded and compared using correlation coefficient and Cohen's Kappa.

Results 162 patients were diagnosed with PanNET in our centre over the study period of which 57 underwent surgical resection (mean age 55.6, 30 males). 22 lesions (mean size $24.5 \mathrm{~mm}$ ) were located in the head, 10 in the body and 25 in the tail of the pancreas. 35 lesions underwent FNA and 26 FNB (4 lesions underwent both) all of which confirmed PanNET on cytology or histology respectively. On surgical histology 33 lesions were grade 1, 22 were grade 2, 1 was grade 3 and 1 was mixed neuroendocrine-acinar. 23/35 FNA samples could report Ki67/grading compared to 26/26 FNB samples $(p=0.0006)$. Ki67 on FNA showed a weak correlation with surgical pathology $(R=-0.08, p=0.74)$ whereas Ki67 on FNB showed a moderate correlation $(\mathrm{R}=0.65, \mathrm{p}=0.0004)$. With respect to tumour grading, FNA samples showed a poor correlation (kappa $=0.026)$ and $\mathrm{FNB}$ samples showed a moderate correlation (kappa=0.474). Excluding cystic lesions gave similar results. 12 samples had been obtained using the Procore needle and 13 samples obtained using the Sharkcore needle. Procore correlation of Ki67 to surgical resection histology was moderate $(\mathrm{r}=0.521,95 \%$ confidence interval $-0.07-0.84$, $\mathrm{p}=0.08)$. Sharkcore correlation of Ki67 to surgical resection histology was good $(r=0.788,95 \%$ confidence interval $0.42-$ $0.93, \mathrm{p}=0.0013)$. With respect to tumour grading, both Procore and Sharkcore showed moderate correlation (kappa $=0.47$ and 0.435 respectively).

Conclusion Both FNA and FNB can be used to confirm a diagnosis of PanNET. However, FNB samples were significantly more likely to provide adequate material for Ki67/grading and showed a closer match to Ki67/grading of the final surgical histology.

\section{OTU-019 PANCREATIC ENZYME SUPPLEMENTATION IS ASSOCIATED WITH IMPROVED SURVIVAL IN INOPERABLE PANCREATIC CANCER}

Rohit Sinha*, Sarah Manning, Charlotte Morrison, John R Greenaway, Debasis Majumdar, Darren G Craig, Syed Zubair, Jeremy Dean, Vikramjit Mitra. James Cook University Hospital, South Tees NHS Foundation Trust, Middlesborough, UK

\subsection{6/gutjnl-2018-BSGAbstracts.305}

Introduction In the UK, $85 \%$ of pancreatic cancer (PC) patients are inoperable at presentation with a median survival of 6-9 months1. BSG guidelines recommend that patients with PC should receive pancreatic enzyme supplementation to maintain bodyweight and improve quality of life 1 . It is not known whether pancreatic enzyme supplementation prolongs survival. In this study, we examined the impact of pancreatic enzyme supplementation in inoperable pancreatic cancer.

Methods This was a single-centre retrospective study between January 2016 and June 2017. Consecutive patients deemed inoperable in the MDT were identified. Patients were subdivided in two cohorts, pancreatic enzyme supplement (PES) and Non-PES. Date of diagnosis was determined by the date of cross-sectional imaging. Use of pancreatic enzymes was determined through patient summary care records. All cases were followed up from the date of diagnosis until the date of death or censor date (31st December 2017). Primary outcomes were all-cause mortality.

Results 62 patients were included (51 had histological confirmation, 11 were radiological diagnosis). Adenocarcinoma was the commonest histological finding. Anatomical distribution of tumours was - head/uncinate (27), neck (5), body (18) and tail (12). MPD dilatation was noted in 33 patients. The mean age among the PES and Non-PES groups were comparable at $70.9( \pm 9.9)$ and $72.2( \pm 9.5)$ years respectively. $48 \%$ were female in PES group compared with $40 \%$ in non-PES group. Median follow up was 133 days (IQR 187). Unadjusted median survival days in PES group was 221 [95\% CI 107.4, 334.6] compared with 61 [95\% CI 35.5, 86.5] in non-PES group. Cox regression models were fitted to adjust for effects of baseline characteristics. Survival was significantly better in PES group (Log Rank $p=0.004$ ) than that seen in non-PES group (figure 1). The survival difference remained significant after adjusting for gender, age, histology of pancreatic cancer, neutrophil to lymphocyte ratio and presenting body-mass index $(n=53)$. Lack of pancreatic enzyme supplementation was associated with significant mortality risk, adjusted hazard ratio of 2.7 [95\% CI 1.38, 5.31; $\mathrm{p}=0.004] .21(78 \%)$ and $17(49 \%)$ patients in PES group $(n=27)$ and non-PES group respectively were treated with palliative chemotherapy and the rest were treated with best supportive care.

Conclusions Our study concludes that pancreatic enzyme supplementation is associated with improved survival in inoperable pancreatic cancer. Further prospective studies are required to confirm our findings. 